\title{
BMJ Open Hyperosmolar therapy for acute brain injury: study protocol for an umbrella review of meta-analyses and an evidence mapping
}

\author{
Rafael Badenes, ${ }^{1,2,3}$ Brian Hutton (D) , , ${ }^{4,5}$ Giuseppe Citerio (D) ,",7 Chiara Robba, ${ }^{8}$ \\ Gerardo Aguilar, ${ }^{1,3}$ Adolfo Alonso-Arroyo, ${ }^{9,10}$ Fabio Silvio Taccone, ${ }^{11}$ \\ Carlos Tornero, ${ }^{1}$ Ferrán Catalá-López (iD) ${ }^{3,5,12,13}$
}

To cite: Badenes R, Hutton B, Citerio G, et al. Hyperosmolar therapy for acute brain injury: study protocol for an umbrella review of meta-analyses and an evidence mapping. BMJ Open 2020;10:e033913. doi:10.1136/ bmjopen-2019-033913

- Prepublication history and additional material for this paper are available online. To view these files, please visit the journal online (http://dx.doi. org/10.1136/bmjopen-2019033913).

Received 27 August 2019 Revised 05 December 2019 Accepted 22 January 2020
Check for updates

(C) Author(s) (or their employer(s)) 2020. Re-use permitted under CC BY-NC. No commercial re-use. See rights and permissions. Published by BMJ.

For numbered affiliations see end of article.

Correspondence to Dr Ferrán Catalá-López; ferran_catala@outlook.com

\section{ABSTRACT}

Introduction Acute brain injury is a challenging public health problem worldwide. Elevated intracranial pressure is a common complication after acute brain injury. Hyperosmolar therapy is one of the main therapeutic strategies for the management of intracranial hypertension. This study protocol outlines an umbrella review of meta-analyses which will investigate the benefits and harms of hyperosmolar therapy routinely used for the management of acute brain injury in the intensive care. Methods and analysis We will search PubMed/ MEDLINE, EMBASE and the Cochrane Database of Systematic Reviews. We will include meta-analyses of primary research studies (eg, randomised controlled trials, observational studies or both) that evaluate one or more hyperosmolar solutions (including hypertonic saline and/or mannitol) for the treatment of adult patients with acute brain injury of any severity. Two researchers will independently screen all citations, full-text articles and abstract data. Potential conflicts will be resolved through discussion with a third researcher. Primary outcomes will be mortality and neurological outcomes at discharge. Secondary outcomes will include control of intracranial pressure, cerebral perfusion pressure, length of stay (in hospital an intensive care unit) and any adverse event. Quality of the included meta-analyses will be assessed using the AMSTAR-2 tool. An overall summary of methods and results will be performed using tabular and graphical approaches and will be supplemented by narrative description. We will analyse whether published meta-analyses present an outline of available evidence (eg, cited, described and discussed any previous metaanalysis). Where objectives from two or more metaanalyses overlap, we will assess the causes of any noted discrepancies between meta-analyses.

Ethics and dissemination No ethical approval will be required. Findings from this study will be published in a peer-reviewed journal. All data will be deposited in a cross-disciplinary public repository.

PROSPERO registration number CRD42019148152.

\section{INTRODUCTION}

Acute brain injury has become a challenging public health problem worldwide, ${ }^{1-3}$
Strengths and limitations of this study

- This will be the first umbrella review to systematically identify and evaluate the quantity, validity and credibility of published meta-analyses on hyperosmolar therapy for acute brain injury.

- Our literature review will be expansive, including meta-analyses of randomised controlled trials, observational studies or both.

- There may be variability in how treatment interventions and outcomes are reported in published meta-analyses, which may pose a challenge in the qualitative (narrative) synthesis.

- There may be a paucity in patient-important outcomes reported in the published meta-analyses testing the benefits and harms of hyperosmolar therapy.

resulting in a large burden of disease in terms of premature death and disability. Complications after acute brain injury cause poor outcomes and enormous losses to individuals, families and communities. Elevated intracranial pressure (ICP) is a common problem in intensive care units (ICUs), being a potentially devastating complication of acute brain injury. Prevention and control of elevated ICP are the cornerstones of treatment for these patients, ${ }^{4-6}$ as uncontrolled hypertension worsens brain damage and remains the most common cause of death after the injury.

Several strategies are recommended for the treatment of elevated ICP in adult patients with acute brain injury. ${ }^{7-9}$ Currently, hyperosmolar therapy with mannitol or hypertonic saline solution is the main therapeutic strategy for the management of intracranial hypertension and cerebral oedema. ${ }^{9}$ Although hyperosmolar therapy has been used extensively in routine clinical practice, ${ }^{10-12}$ significant 
controversy regarding the choice of hyperosmolar agent and dosing exists.

Systematic reviews with meta-analyses are prominent tools to guide healthcare decisions and inform research agendas. A growing number of systematic reviews and meta-analyses aiming to evaluate the effects of hyperosmolar solutions in acute brain injury have been published showing inconsistent results. ${ }^{13-23}$ As the number of published meta-analyses increases, conflicts and divergent conclusions among them can emerge producing difficulties for decision makers who rely on these studies. ${ }^{24}$ In addition, many meta-analysis authors may not discuss the results of previous meta-analyses and systematic reviews. ${ }^{25}$ This situation can be confusing for readers and knowledge users, may contribute to unnecessary duplications of meta-analysis ${ }^{26-28}$ and possibly cause waste in research planning, conduct and reporting. ${ }^{29} 30$

This study has been designed to answer the following primary research question: what is the effectiveness and safety of hyperosmolar therapy (mannitol and hypertonic saline) routinely used for the management of acute brain injury in intensive care? There are three aims within the planned study. The first aim will be to evaluate the quantity, validity and credibility of the evidence from existing systematic reviews and meta-analyses with regard to the benefits and harms of hyperosmolar therapy for acute brain injury. The second aim will be to provide a robust assessment of the evidence in this area using a revised and improved quality assessment tool: AMSTAR-2. ${ }^{31}$ The third aim will be to investigate whether published metaanalyses present an outline of available evidence by referring to previous meta-analyses and systematic reviews, and compare and contrast findings across overlapping metaanalyses on the same topic.

\section{METHODS AND ANALYSIS}

This review protocol is part of an evidence synthesis project on the comparative effectiveness and safety of hyperosmolar therapy in neurocritical care. We will conduct an umbrella review (or 'overview of reviews'), ${ }^{32} 33$ which is the systematic collection and assessment of multiple systematic reviews and meta-analyses carried out in a specific research topic. This study protocol has been registered within PROSPERO database (registration number: CRD42019148152), the Open Science Framework (https://osf.io/mcqpv), and has been prepared in consultation with the reporting guidance in the Preferred Reporting Items for Systematic Reviews and Meta-Analyses Protocols ${ }^{34} 35$ (see online supplementary appendix 1 for the checklist). Methods have been chosen in consultation with methodological work, ${ }^{32-41}$ including the Cochrane Handbook's chapter on methods for 'overviews of reviews'. 33

\section{Eligibility criteria}

Detailed eligibility criteria have been developed following the population, interventions, comparators, outcomes and study design format and are described below.

\section{Population}

This study will include adult patients over the age of 18 years admitted to an ICU with an acute primary neurological diagnosis (that is, traumatic brain injury, stroke or intracerebral haemorrhage) of any severity. Patients with disseminated cerebral disorders (such as meningitis, encephalitis, acute hepatic failure and neoplasms) will be excluded.

\section{Interventions and comparators}

The intervention group will be intravenous administration of hyperosmolar therapy routinely used in the ICU, including hypertonic saline at any dose (eg, $3 \%, 7.5 \%$ or $23.4 \%$ sodium chloride) and mannitol at any dose (e.g. $\geq 20 \%$ mannitol) for any duration. The control group could be placebo-controlled, standard of care, no therapy and different therapy (eg, alternative fluid therapy such as $0.9 \%$ sodium chloride (normal saline), $\leq 0.45 \%$ sodium chloride (hypotonic saline), $5 \%$ dextrose, Ringer's lactate, Hartmann's solution). Treatment comparisons of interest will include hyperosmolar therapy in monotherapy and/ or combination therapy regimens against each other or against an alternative control group.

\section{Outcomes}

Results on all primary and secondary outcomes of the included systematic reviews and meta-analyses will be reported. However, we will define the following primary and secondary outcomes for this study. The primary outcomes will be patient-important outcomes including mortality from all causes (defined as 90 day mortality, if not available, 30-day ICU or hospital mortality, whichever was longest) and neurological outcomes at discharge (as reported by authors of included studies, eg, Glasgow Outcome Scale, extended Glasgow Outcome Scale or modified Rankin Scale). The secondary outcomes will be ICP $(\mathrm{mm} \mathrm{Hg})$, cerebral perfusion pressure $(\mathrm{mm} \mathrm{Hg})$, any adverse events (as reported by authors of included studies) and important health services use measures such as length of hospital stay and length of ICU stay (both in days).

\section{Study design}

We will consider published articles of completed systematic reviews with meta-analysis of randomised controlled trials, observational studies (eg, cohort studies, casecontrol studies) or both. We will include articles that explicitly stated methods to identify studies (eg, a search strategy), explicitly stated methods of study selection (eg, eligibility criteria and selection) and explicitly described methods of quantitative data synthesis (eg, at least reported a meta-analysis summarising the results of several studies into a single estimate). To ensure as broad a scope of content as possible, all reviews with 
meta-analysis, including updates of previous reviews with meta-analyses, and reviews with overlapping evidence base (eg, primary studies could appear within more than one included meta-analysis) will be eligible for inclusion. Only references published in English will be eligible as the vast majority of meta-analyses are published in this language. Meta-analyses not reporting a systematic methodology and narrative reviews will be excluded.

\section{Searching}

We will search PubMed/MEDLINE, EMBASE and the Cochrane Database of Systematic Reviews (from January 2000 onwards-considering the first Cochrane review on mannitol for acute traumatic brain injury was published in $2000^{13}$ ). Intended date for the literature searches in PubMed/MEDLINE, EMBASE and the Cochrane Database of Systematic Reviews will be 15 May 2020. The literature searches will be conducted by an experienced information specialist. The main literature search will be peer-reviewed by a senior health information specialist using the Peer Review of Electronic Search Strategies checklist. ${ }^{42}$ The draft search strategy can be found in online supplementary appendix 2. To ensure literature saturation, we will scan the reference lists of included meta-analyses, and relevant reviews and clinical practice guidelines identified through the search.

\section{Screening and selection procedure}

All articles identified from the literature search will be screened by two researchers independently. First, titles and abstracts of articles returned from initial searches will be screened based on the eligibility criteria outlined earlier. Second, full texts will be examined in detail and screened for eligibility. Third, references of all considered articles will be hand-searched to identify any relevant report missed in the search strategy. Any discrepancies here and throughout will be resolved through consensus or recourse to a third researcher, if necessary. A flow chart showing details of studies included and excluded at each stage of the study selection process will be provided.

\section{Data collection}

Data for each of the included meta-analysis will be abstracted by two researchers, independently, and potential conflicts will be resolved through discussion. We will use pre-designed forms that will be piloted initially on a small number of included articles. The data extracted from each article will be comprehensive in scope as we are addressing multiple review objectives. Information of interest will include the following: first author, year of publication, reported a protocol, objective(s), reported strategies to search literature, number of databases searched and date of last search, any restrictions (eg, language, geographic, or date), inclusion/exclusion criteria, intervention(s) of interest and comparators, patient population, main outcomes of interest, type of study designs included (eg, randomised controlled trials, observational studies, or both), number of included studies, number of studies reporting data for metaanalyses, effect metric(s) reported (eg, risk ratio, risk difference, standardised mean difference), methods to assess study quality/risk of bias, statistical methods to combine studies, summary meta-analytic estimates including heterogeneity measures, additional analyses (eg, subgroup analysis or sensitivity analysis), metabias assessment (eg, publication bias across studies), funding source and conflicts of interest, whether a previous metaanalysis was cited (eg, whether a reference to the earlier article was provided as part of the background and/ or rationale for the study), described (eg, whether any information about the results of the earlier article was provided) and discussed (eg, the results from the earlier article were related to the results or conclusions from the most recent study) by the published meta-analysis.

\section{Quality assessment of meta-analyses}

The methodological quality of meta-analyses will be evaluated using the AMSTAR-2 tool (see online supplementary appendix 3 for the checklist). This revised version of the original AMSTAR tool ${ }^{434}$ allows for the appraisal of systematic reviews and meta-analyses of randomised and non-randomised studies of healthcare interventions. ${ }^{31}$ We will evaluate each included meta-analysis against the 16 items of the tool, and an overall rating will be assigned. Briefly, the AMSTAR-2 tool considers the following seven domains that can critically affect the validity of a meta-analysis and its conclusions: registration of the protocol prior to commencement of the review, adequacy of the literature search, justification for excluding individual studies, assessment of risk of bias of included studies, appropriateness of the meta-analytic methods, consideration of risk of bias when interpreting results and assessment of presence and impact of publication bias. Meta-analyses failing to meet any of the critical domains will be deemed to have a 'critical flaw' while non-fulfilment of the remaining items will be deemed a 'non-critical weakness'. Meta-analyses with no 'critical flaws' will be considered either high or moderate quality depending on the number of 'non-critical weaknesses' (eg, high-quality meta-analyses have a maximum of one 'non-critical weaknesses' and moderate-quality metaanalyses have more than one). Meta-analyses with one or more 'critical flaws' will receive a low or very low rating, respectively. The quality of meta-analyses will be evaluated by one researcher and verified by another. Disagreements regarding quality ratings will be resolved by discussion with a third researcher.

\section{Data analysis}

To summarise findings, a descriptive approach will be taken that will include tables to characterise key features, findings, and variations of the research, supplemented with graphics (eg, funnel plots) to highlight diversity in study results and other aspects. Guidance from the Cochrane Handbook will be followed. ${ }^{33}$ Because multiple meta-analyses may include information from 
overlapping individual studies, we will not perform a separate meta-analysis of pooled effect estimates. Instead, we will perform a systematic narrative (qualitative) synthesis or summary for each outcome and intervention reporting the pooled treatment effects from the most comprehensive and highest-quality meta-analyses (eg, high and moderate quality meta-analyses as assessed by the AMSTAR-2 approach ${ }^{31}$ ). We will provide a narrative summary of the quality assessment of the included metaanalyses, which will be supported by a table showing the results of the critical appraisal. In addition, the narrative synthesis will explore the relationship and findings both within and between the included meta-analyses.

In exploring the rationale for variations or discrepancies in findings between published meta-analyses, several strategies will be employed. First, comparison of methods will be performed in relation to eligibility criteria (eg, assessment of potential differences in criteria used to identify eligible patients, study designs and outcomes), literature search details (eg, databases, dates and key differences in search strategies), outcome definitions used, statistical approaches to meta-analysis and rigour of methods (as reflected by potential variations in AMSTAR-2 assessments ${ }^{31}$ ). Second, the evidence base included in different meta-analyses and systematic reviews will be mapped in terms of their degree of overlap. This will involve comparison of date ranges of studies covered, the numbers of studies and patients across meta-analyses and development of a citation matrix to establish the similarity of included study lists. Third, comparison of meta-analysis findings and conclusions drawn by research teams will also be performed.

\section{Subgroup and sensitivity analyses}

In addition to the previously identified pre-planned analyses, we will examine clinical heterogeneity with primary neurological diagnosis (eg, ischaemic vs traumatic brain injury), disease severity (eg, moderate and severe acute brain injury) and hyperosmolar therapy when given in different doses and/or for different durations (eg, continuous hyperosmolar therapy vs any use). ${ }^{45} 46$ If data permit, we will also explore methodological heterogeneity with type of study designs included (eg, randomised controlled trials, observational studies or both).

\section{Confidence in cumulative evidence}

We will use the Grading of Recommendations Assessment, Development and Evaluation approach ${ }^{47}{ }^{48}$ to rate the overall quality of the evidence. We will create an adapted summary of findings table based on the methods described in the Cochrane Handbook ${ }^{33}$ to convey key information about the effect for each treatment and overall confidence in the treatment of effect estimates.

\section{Patient and public involvement}

There has been no patient and public involved in setting the research question or developing plans for design (or implementation) of this study protocol.

\section{ETHICS AND DISSEMINATION}

This manuscript outlines a study protocol for an umbrella review that will undertake secondary data analysis and hence does not require ethical approval. The purpose of this study will be to provide an evidence synthesis to identify which hyperosmolar solutions for treating acute brain injury would be supported by most convincing evidence. The study findings will be disseminated through presentations at clinical conferences and publication in a peerreviewed journal. Any amendments made to this protocol when conducting the analyses will be outlined and reported in the final manuscript. All data underlying the findings reported in the final manuscript will be deposited in a cross-disciplinary public repository such as the Open Science Framework (https://osf.io/mcqpv).

\section{Author affiliations}

${ }^{1}$ Department of Anesthesiology and Surgical-Trauma Intensive Care, Hospital Clinic Universitari de València, University of Valencia, Valencia, Spain

${ }^{2}$ Department of Surgery, Faculty of Medicine, University of Valencia, Valencia, Spain ${ }^{3}$ INCLIVA Health Research Institute, Valencia, Spain

${ }^{4}$ School of Epidemiology and Public Health, University of Ottawa, Ottawa, Ontario, Canada

${ }^{5}$ Clinical Epidemiology Program, Ottawa Hospital Research Institute, Ottawa, Ontario, Canada

${ }^{6}$ Neurointensive Care, San Gerardo Hospital, ASST-Monza, Monza, Italy ${ }^{7}$ School of Medicine and Surgery, University Milano Bicocca, Milan, Italy ${ }^{8}$ Department of Anesthesia and Intensive Care, IRCCS Policlinico San Martino, Genoa, Italy

${ }^{9}$ Department of History of Science and Documentation, University of Valencia, Valencia, Spain

${ }^{10}$ Information and Social and Health Research Unit (UISYS), University of Valencia and Spanish National Research Council (CSIC), Valencia, Spain

${ }^{11}$ Department of Intensive Care, Erasme Hospital, Université Libre de Bruxelles, Brussels, Belgium

${ }^{12}$ Department of Health Planning and Economics, National School of Public Health, Institute of Health Carlos III, Madrid, Spain

${ }^{13}$ Department of Medicine, Faculty of Medicine, University of Valencia/CIBERSAM, Valencia, Spain

\section{Twitter Giuseppe Citerio @dr_Cit}

Contributors All authors contributed to conceptualising and designing the study. FC-L and RB drafted the manuscript. BH, GC, CR, GA, AA-A, FT and CT commented for important intellectual content and made revisions. All authors read and approved the final version of the manuscript. RB and FC-L accept full responsibility for the finished manuscript and controlled the decision to publish.

Funding FC-L is supported by the Institute of Health Carlos III/CIBERSAM. BH is supported by a New Investigator Award from the Canadian Institutes of Health Research and the Drug Safety and Effectiveness Network. The funders were not involved in the design of the protocol or decision to submit the protocol for publication, nor will they be involved in any aspect of the study conduct. The views expressed in this manuscript are those of the authors and many not be understood or quoted as being made on behalf of, or reflecting the position of, the funder(s) or any institution.

Competing interests None declared.

Patient consent for publication Not required.

Provenance and peer review Not commissioned; externally peer reviewed.

Data availability statement Data sharing not applicable as no data sets generated and/or analysed for this study.

Open access This is an open access article distributed in accordance with the Creative Commons Attribution Non Commercial (CC BY-NC 4.0) license, which permits others to distribute, remix, adapt, build upon this work noncommercially, and license their derivative works on different terms, provided the original work is properly cited, appropriate credit is given, any changes made 
indicated, and the use is non-commercial. See: http://creativecommons.org/ licenses/by-nc/4.0/.

\section{ORCID iDs}

Brian Hutton http://orcid.org/0000-0001-5662-8647

Giuseppe Citerio http://orcid.org/0000-0002-5374-3161

Ferrán Catalá-López http://orcid.org/0000-0002-3833-9312

\section{REFERENCES}

1 Feigin VL, Nichols E, Alam T, et al. Global, regional, and national burden of neurological disorders, 1990-2016: a systematic analysis for the global burden of disease study 2016. Lancet Neurol 2019;18:459-80.

2 James SL, Theadom A, Ellenbogen RG, et al. Global, regional, and national burden of traumatic brain injury and spinal cord injury, 19902016: a systematic analysis for the global burden of disease study 2016. Lancet Neurol 2019;18:56-87.

3 Maas AIR, Menon DK, Adelson PD, et al. Traumatic brain injury: integrated approaches to improve prevention, clinical care, and research. Lancet Neurol 2017;16:987-1048.

4 Fernando SM, Tran A, Cheng W, et al. Diagnosis of elevated intracranial pressure in critically ill adults: systematic review and meta-analysis. BMJ 2019;366:14225.

5 Stocchetti N, Maas AIR. Traumatic intracranial hypertension. N Engl J Med 2014:370:2121-30.

6 Ropper $\mathrm{AH}$. Hyperosmolar therapy for raised intracranial pressure. $\mathrm{N}$ Engl J Med 2012;367:746-52.

7 Carney N, Totten AM, O'Reilly C, et al. Guidelines for the management of severe traumatic brain injury, fourth edition. Neurosurgery 2017;80:6-15.

8 Stocchetti N, Carbonara M, Citerio G, et al. Severe traumatic brain injury: targeted management in the intensive care unit. Lancet Neurol 2017;16:452-64.

9 Oddo M, Poole D, Helbok R, et al. Fluid therapy in neurointensive care patients: ESICM consensus and clinical practice recommendations. Intensive Care Med 2018;44:449-63.

10 Hays AN, Lazaridis C, Neyens R, et al. Osmotherapy: use among neurointensivists. Neurocrit Care 2011;14:222-8.

11 Berger Pelletier E, Émond M, Lauzier F, et al. Hyperosmolar therapy in severe traumatic brain injury: a survey of emergency physicians from a large Canadian Province. PLoS One 2014;9:e95778.

12 Wijayatilake DS, Talati C, Panchatsharam S. The monitoring and management of severe traumatic brain injury in the United Kingdom: is there a consensus?: a national survey. J Neurosurg Anesthesiol 2015;27:241-5.

13 Roberts I, Schierhout G, Wakai A, et al. Mannitol for acute traumatic brain injury. Cochrane Database Syst Rev 2003;2.

14 Barbic D, Barbic S, Lang E, et al. Hypertonic saline in acute traumatic brain injury? A systematic review and meta analysis. CJEM 2010;12:231.

15 Kamel H, Navi BB, Nakagawa K, et al. Hypertonic saline versus mannitol for the treatment of elevated intracranial pressure: a metaanalysis of randomized clinical trials. Crit Care Med 2011;39:554-9.

16 Mortazavi MM, Romeo AK, Deep A, et al. Hypertonic saline for treating raised intracranial pressure: literature review with metaanalysis. J Neurosurg 2012;116:210-21.

17 Wakai A, McCabe A, Roberts I, et al. Mannitol for acute traumatic brain injury. Cochrane Database Syst Rev 2013;3.

18 Rickard AC, Smith JE, Newell P, et al. Salt or sugar for your injured brain? A meta-analysis of randomised controlled trials of mannitol versus hypertonic sodium solutions to manage raised intracranial pressure in traumatic brain injury. Emerg Med J 2014;31:679-83.

19 Wang K, Sun M, Jiang H, et al. Mannitol cannot reduce the mortality on acute severe traumatic brain injury (TBI) patients: a meta-analyses and systematic review. Burns Trauma 2015;3:1-8.

20 Berger-Pelleiter E, Émond M, Lauzier F, et al. Hypertonic saline in severe traumatic brain injury: a systematic review and meta-analysis of randomized controlled trials. CJEM 2016;18:112-20.

21 Burgess S, Abu-Laban RB, Slavik RS, et al. A systematic review of randomized controlled trials comparing hypertonic sodium solutions and mannitol for traumatic brain injury: implications for emergency department management. Ann Pharmacother 2016;50:291-300.

22 Poole D, Citerio G, Helbok R, et al. Evidence for mannitol as an effective agent against intracranial hypertension: an individual patient data meta-analysis. Neurocrit Care 2019;312.

$23 \mathrm{Gu}$ J, Huang $\mathrm{H}$, Huang $\mathrm{Y}$, et al. Hypertonic saline or mannitol for treating elevated intracranial pressure in traumatic brain injury: a meta-analysis of randomized controlled trials. Neurosurg Rev 2019;42:499-509.

24 Jadad AR, Cook DJ, Browman GP. A guide to interpreting discordant systematic reviews. CMAJ 1997;156:1411-6.

25 Helfer B, Prosser A, Samara MT, et al. Recent meta-analyses neglect previous systematic reviews and meta-analyses about the same topic: a systematic examination. BMC Med 2015;13:82

26 Moher D. The problem of duplicate systematic reviews. BMJ 2013;347:f5040.

27 Siontis KC, Hernandez-Boussard T, loannidis JPA. Overlapping meta-analyses on the same topic: survey of published studies. BMJ 2013;347:f4501.

28 Naudet F, Schuit E, loannidis JPA. Overlapping network metaanalyses on the same topic: survey of published studies. Int $J$ Epidemiol 2017;46:1999-2008.

29 Chalmers I, Bracken MB, Djulbegovic B, et al. How to increase value and reduce waste when research priorities are set. The Lancet 2014;383:156-65.

30 loannidis JPA, Greenland S, Hlatky MA, et al. Increasing value and reducing waste in research design, conduct, and analysis. The Lancet 2014;383:166-75.

31 Shea BJ, Reeves BC, Wells G, et al. AMSTAR 2: a critical appraisal tool for systematic reviews that include randomised or nonrandomised studies of healthcare interventions, or both. BMJ 2017;358:j4008.

32 Ioannidis JPA. Integration of evidence from multiple meta-analyses: a primer on umbrella reviews, treatment networks and multiple treatments meta-analyses. Can Med Assoc J 2009;181:488-93.

33 Becker LA, Oxman A. Chapter 22: Overviews of reviews. In: Cochrane Handbook for systematic reviews of interventions, version 5.1.0, 2011. https://handbook-5-1.cochrane.org/chapter_22/22_ overviews_of_reviews.htm

34 Moher D, Shamseer L, Clarke M, et al. Preferred reporting items for systematic review and meta-analysis protocols (PRISMA-P) 2015 statement. Syst Rev 2015;4.

35 Shamseer L, Moher D, Clarke M, et al. Preferred reporting items for systematic review and meta-analysis protocols (PRISMA-P) 2015: elaboration and explanation. BMJ 2015;349:97647.

36 Smith V, Devane D, Begley CM, et al. Methodology in conducting a systematic review of systematic reviews of healthcare interventions. BMC Med Res Methodol 2011;11:15.

37 Hunt $\mathrm{H}$, Pollock A, Campbell P, et al. An introduction to overviews of reviews: planning a relevant research question and objective for an overview. Syst Rev 2018;7:39.

38 Aromataris E, Fernandez R, Godfrey C. Chapter 10: Umbrella Reviews. In: Aromataris E, Munn Z, eds. Joanna Briggs Institute reviewer's manual. Adelaide: The Joanna Briggs Institute, 2017. https://wiki.joannabriggs.org/display/MANUAL/Chapter+10\%3A+ Umbrella+reviews

39 Aromataris E, Fernandez R, Godfrey CM, et al. Summarizing systematic reviews: methodological development, conduct and reporting of an umbrella review approach. Int $J$ Evid Based Healthc 2015;13:132-40.

40 Bougioukas KI, Liakos A, Tsapas A, et al. Preferred reporting items for overviews of systematic reviews including harms checklist: a pilot tool to be used for balanced reporting of benefits and harms. J Clin Epidemiol 2018;93:9-24.

41 Lunny C, Brennan SE, Reid J, et al. Overviews of reviews incompletely report methods for handling overlapping, discordant, and problematic data. J Clin Epidemiol 2019;118:69-85.

42 McGowan J, Sampson M, Salzwedel DM, et al. PRESS Peer Review of Electronic Search Strategies: 2015 Guideline Statement. J Clin Epidemiol 2016;75:40-6.

43 Shea BJ, Grimshaw JM, Wells GA, et al. Development of AMSTAR: a measurement tool to assess the methodological quality of systematic reviews. BMC Med Res Methodol 2007;7:10.

44 Shea BJ, Hamel C, Wells GA, et al. AMSTAR is a reliable and valid measurement tool to assess the methodological quality of systematic reviews. J Clin Epidemiol 2009;62:1013-20.

45 Asehnoune K, Lasocki S, Seguin P, et al. Association between continuous hyperosmolar therapy and survival in patients with traumatic brain injury - a multicentre prospective cohort study and systematic review. Crit Care 2017;21:328.

46 Ichai C, Payen J-F, Orban J-C, et al. Half-molar sodium lactate infusion to prevent intracranial hypertensive episodes in severe traumatic brain injured patients: a randomized controlled trial. Intensive Care Med 2013;39:1413-22.

47 Atkins D, Best D, Briss PA, et al. Grading quality of evidence and strength of recommendations. BMJ 2004;328:1490. 
